\title{
Substitution Pattern Controlled Quantum Interference in [2.2]Paracyclophane-Based Single-Molecule Junctions
}

\author{
Ksenia Reznikova, ${ }^{\#}$ Chunwei Hsu, ${ }^{\#}$ Werner M. Schosser, Almudena Gallego, Katawoura Beltako, \\ Fabian Pauly,* Herre S. J. van der Zant,* and Marcel Mayor*
}

Cite This: J. Am. Chem. Soc. 2021, 143, 13944-13951

Read Online

ACCESS |

山ll Metrics \& More

回 Article Recommendations

Supporting Information

ABSTRACT: Quantum interference (QI) of electron waves passing through a single-molecule junction provides a powerful means to influence its electrical properties. Here, we investigate the correlation between substitution pattern, conductance, and mechanosensitivity in [2.2] paracyclophane (PCP)-based molecular wires in a mechanically controlled break junction experiment. The effect of the meta versus para connectivity in both the central PCP core and the phenyl ring connecting the terminal anchoring group is studied. We find that the meta-phenyl-anchored PCP yields such low conductance levels that molecular features cannot be resolved; in the case of para-phenyl-coupled anchoring, however, large variations in conductance values for modulations of the electrode separation
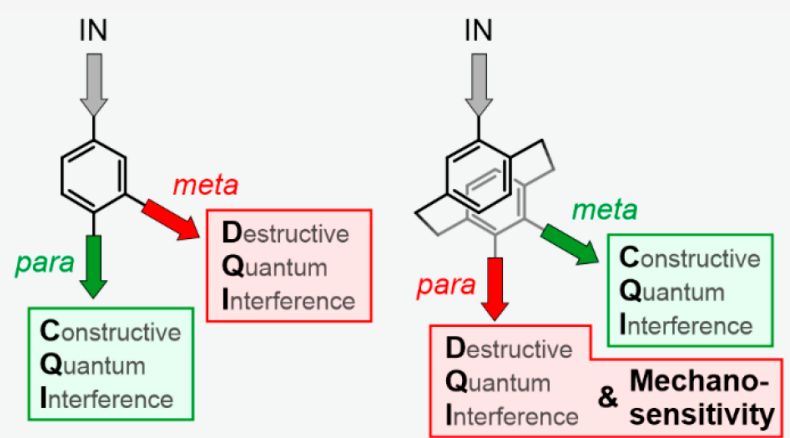
occur for the pseudo-para-coupled PCP core, while this mechanosensitivity is absent for the pseudo-meta-PCP core. The experimental findings are interpreted in terms of QI effects between molecular frontier orbitals by theoretical calculations based on density functional theory and the Landauer formalism.

\section{INTRODUCTION}

In recent years, great advancements have been made in the field of molecular electronics toward single-molecule junction studies. ${ }^{1}$ The visionary idea of Aviram and Ratner ${ }^{2}$ to profit from single molecules as functional units in electronic devices led to the development of several proof-of-concept molecular devices, such as molecular wires, ${ }^{3,4}$ switches, ${ }^{5,6}$ rectifiers/ diodes, ${ }^{7,8}$ and thermo-electronic devices. ${ }^{9,10}$ The design of molecules incorporated in such electrode-molecule-electrode junctions is guided by our understanding of charge transport through the molecules. Indeed, even small structural modifications such as substituent effects, ${ }^{11,12}$ conformational flexibility, ${ }^{13}$ and changes in the anchoring groups and their positions $^{14-18}$ can result in large conductance variations. Particularly strong variations are predicted for quantum interference (QI) effects originating from the interplay between different transport pathways. Destructive QI (DQI) or constructive QI (CQI) between the pathways can occur, reflected in a low or high conductance, respectively. ${ }^{19}$ QI effects thus become essential molecular design elements, on the one hand enriching the variety of functionalities emerging from the molecular structure but on the other hand making a full comprehension of the molecule's electronic transport behavior more challenging. A detailed understanding of these QI effects and of their origin in the molecule's structure is thus crucial to realize their full potential in future electronic components and devices.
Relationships between substitution pattern and singlemolecule conductance were already theoretically predicted and experimentally confirmed in a variety of examples, ranging from simple phenyl rings ${ }^{20}$ connected directly to the electrodes to more sophisticated oligo(phenylene vinylene) (OPV $)^{21}$ and oligo(phenylene ethynylene) (OPE)-based molecular wires. ${ }^{15}$ Unanimously, these studies report a decrease in electronic transparency upon shifting the anchoring groups from the para to the meta position. This observation was rationalized by Yoshizawa and co-workers, ${ }^{22-24}$ who considered frontier orbital theory for simple organic molecules. Their set of rules predicts for benzene the para connection to be the symmetry-allowed one for charge transport, while charge transport involving the meta connection is symmetryforbidden, resulting in high and low conductance, respectively.

Because the effects of substitution patterns in planar $\pi$ systems are well described and understood, our focus moved to three-dimensional structures like [2.2] paracyclophane (PCP), with two benzene systems facing each other interlinked by a pair of $\mathrm{C}_{2} \mathrm{H}_{4}$ bridges. ${ }^{25}$ Initially, we considered the structure as

Received: July 5, 2021

Published: August 23, 2021 
a model to investigate the through-space coupling of the stacked $\pi$-systems ${ }^{26,27}$ but realized quickly that their behavior is much richer. Already the first model compound ${ }^{28}$ (ps-parapara-OPE PCP in Figure 1) displayed a sharp destructive QI
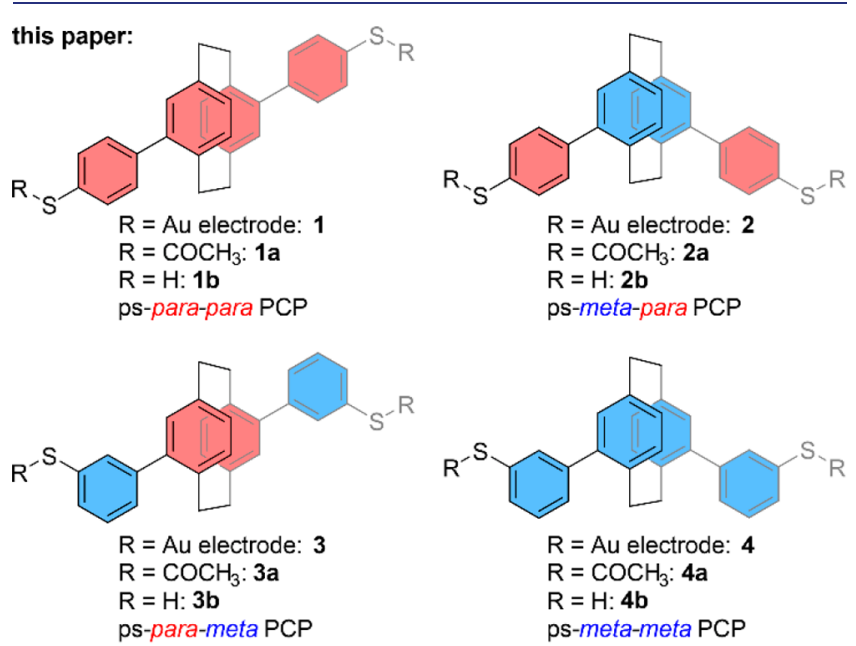

previous work:

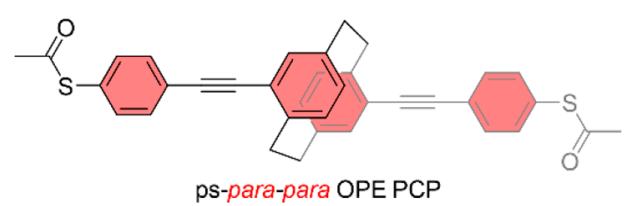

Figure 1. Schematic representation of target structures 1-4 together with the already reported model compound. The four molecules include either para or meta connection patterns in both the central PCP subunit and the peripheral phenyl subunits labeled in red and blue, respectively. For simplicity, the following text refers to the structures by their prefixes, with ps as an abbreviation for pseudo, followed by the prefix referring to the substitution pattern of the central PCP, and finally the prefix referring to the substitution pattern of the thiol anchor group in the phenyl subunits.

feature close to the Fermi level, but the phenomenon also depended substantially on the mechanical stress exposed to the molecule. It thus seemed that the rigid but squeezable PCP subunit provided mechanosensitivity to the molecular junction. Interestingly, Yoshizawa and co-workers ${ }^{29}$ already provided their orbital view of PCP subunits and predicted that the charge transport through the pseudo-para-substituted PCP should be suppressed due to DQI, while CQI would prevail for pseudo-meta-substituted PCP. It thus seems that the rule-of- thumb (para $\rightarrow$ good transport due to CQI; meta $\rightarrow$ bad transport due to DQI) is inverted for the PCP subunit.

Excited by this hypothesis, we explored the effects of the substitution pattern in more detail and designed the four PCP model compounds 1-4 (see Figure 1) consisting of comparable subunits but with various substitution patterns. The oligo(phenylene)-type PCP structures combine the compactness favoring electronic transparency on a detectable level with straightforward synthetic accessibility. Terminal acetyl masked thiol anchor groups enable their immobilization in a mechanically controlled break junction (MCBJ) by covalent $\mathrm{S}-\mathrm{Au}$ bonds, guaranteeing both electronic coupling and mechanical stability. The latter is of particular importance to enable subtle mechanical manipulation of the integrated single molecule. The investigation of their transport properties and the influence of mechanical manipulations are studied with MCBJ experiments. The findings are rationalized by QI effects emerging from the interplay of frontier molecular orbitals, discussed with a theoretical model based on density functional theory (DFT). The electronic transport is described in terms of the Landauer formalism, ${ }^{30}$ expressed through nonequilibrium Green's function (NEGF) methods.

\section{RESULTS AND DISCUSSION}

The PCP-based model compounds were assembled from the corresponding para/meta building blocks by Suzuki-Miyaura cross-coupling reactions. The literature known pseudo (ps)para- or ps-meta-dibromo[2.2] paracyclophane ${ }^{31}$ and either 4or 3-(tert-butylthio)phenyl boronic acid provided the PCP derivatives with four different combinations of substitution patterns. The adaption of a protocol from Jevric et al. ${ }^{32}$ enabled the subsequent transprotection and provided the target structures $\mathbf{1 a - 4 a}$ in reasonable isolated yields ranging from 63 to $85 \%$. The identity of the new PCP derivatives was corroborated by ${ }^{1} \mathrm{H}$ NMR, ${ }^{13} \mathrm{C}\left\{{ }^{1} \mathrm{H}\right\}$ NMR spectroscopy, and high-resolution mass spectrometry (HR-MS). A detailed description of the synthetic protocols and the analytical data of all new compounds are provided in the Supporting Information (section 1).

The single-molecule electronic transport properties of PCPs 1-4 were investigated by integrating them into an electronic circuit using an MCBJ setup operated at ambient conditions. Two types of measurements were performed: fast-breaking and modulation experiments (see below). Details of the technique providing a pair of mechanically adjustable electrodes with a distance resolution of atomic dimensions have been reported
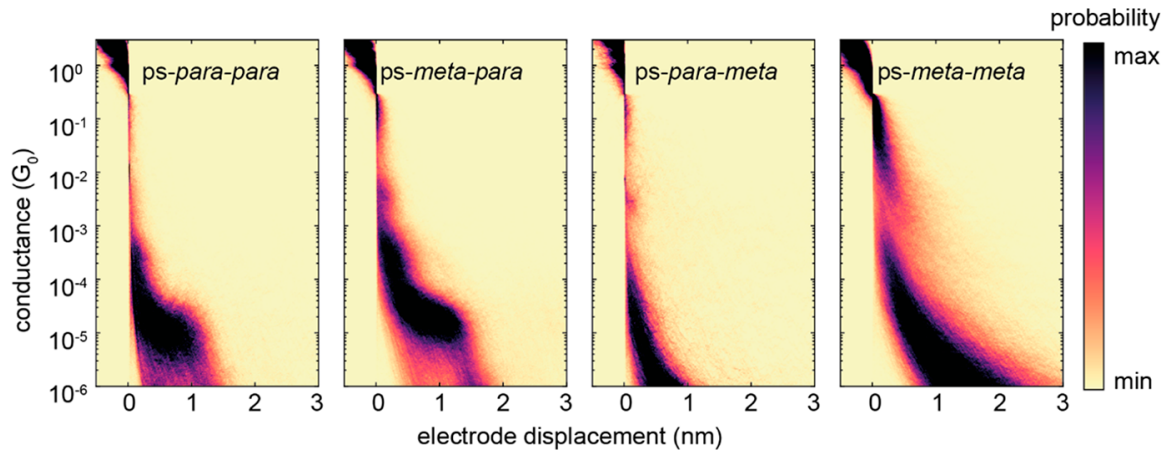

Figure 2. Two-dimensional conductance vs electrode displacement density histograms. The first (from the left) and second histograms are built up from 6834 and 9638 traces at $100 \mathrm{mV}$ with ps-para-para- and ps-meta-para-PCP molecules, respectively. The third and fourth 2D histograms correspond to 3780 and 10000 traces at a bias voltage at $250 \mathrm{mV}$ with ps-para-meta- and ps-meta-meta-PCP molecules, respectively. 

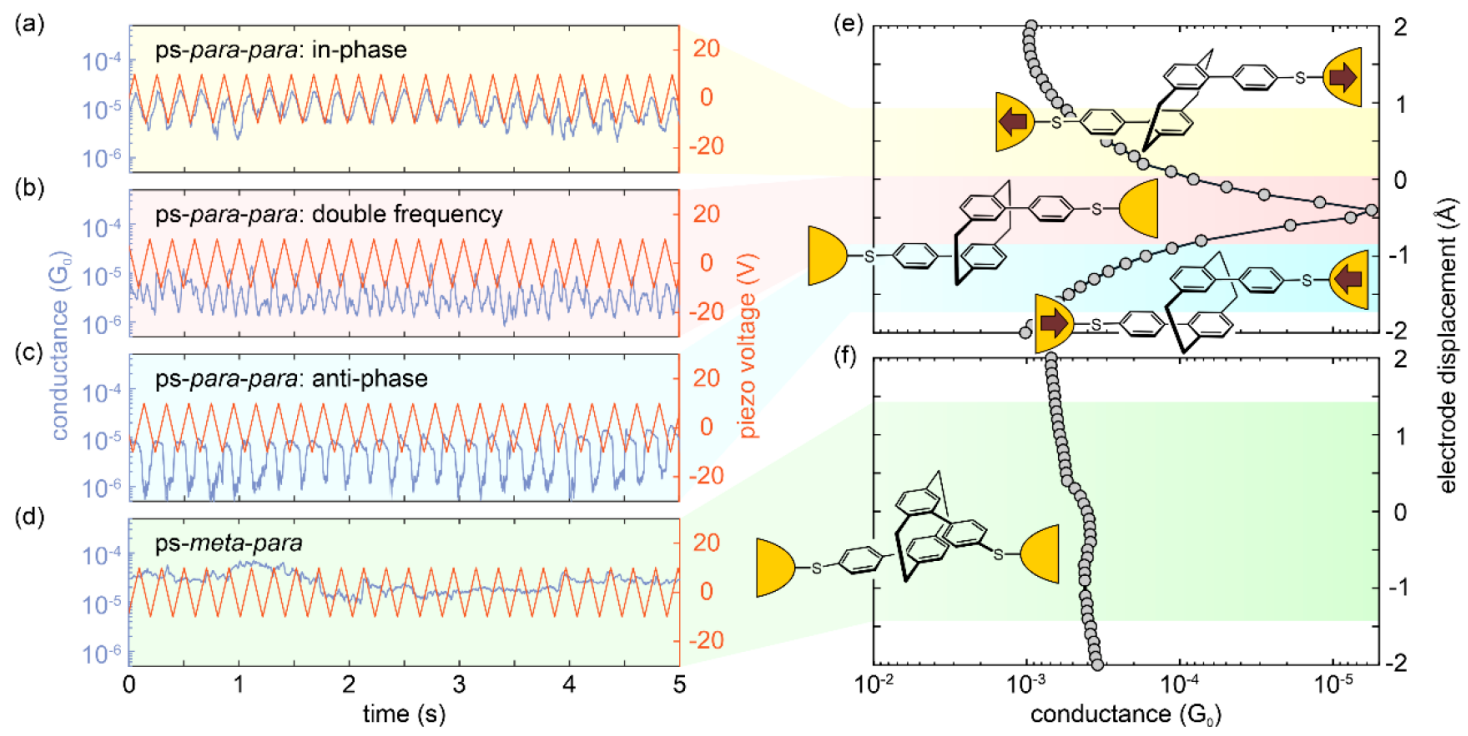

Figure 3. $(\mathrm{a}-\mathrm{c})$ Conductance traces of modulation experiments with ps-para-para-PCP (1), displaying (a) in-phase, (b) double frequency, and (c) anti-phase modulations. (d) Conductance traces of the modulation experiments with ps-meta-para-PCP (2). The red line in the distancemodulation traces represents the voltage applied to the piezoelectric stack, whereas blue represents the measured conductance. The total modulation time of the experiments is $15 \mathrm{~s}$; for better visibility, only $5 \mathrm{~s}$ are displayed. (e) Calculated conductance of the ps-para-para-PCP molecular junction during the gap opening. The conductance displacement data is extracted from Figure $5 \mathrm{~b}$ by evaluating the transmission function at the Fermi energy. The sketches rationalize the variety of conductance vs electrode displacement modulation behaviors observed for ps-para-paraPCP in dependence of the position of the DQI dip with respect to the trapping state of the molecule in the individual junction; see panels a-c and e. In particular, light background colors (yellow, red, and blue) relate the different situations of junctions, including molecules in prestretched, relaxed, or precompressed states, respectively, to the behavior in modulation experiments. (f) Same plot as in panel e but for ps-meta-para-PCP, where mechanosensitivity is basically absent. The green background color connects the conductance that is rather insensitive to electrode displacements to the observations made in the modulation measurements in panel $\mathrm{d}$.

previously $^{33,34}$ and are thus discussed in the Supporting Information (section 2.1).

In the fast-breaking experiments, several thousand conductance traces for each investigated molecule were collected and plotted as two-dimensional (2D) histograms displayed in Figure 2. For the case of ps-para-para- and ps-meta-para-PCPs with a constant bias voltage of $100 \mathrm{mV}$, clear conductance plateaus with a length of $\sim 12.5 \AA$ were observed. Through a reference-free clustering method ${ }^{35}$ on the unfiltered data, the pure gold-to-gold tunneling traces and the molecular traces were separated. The molecular conductances of ps-para-paraand ps-meta-para-PCPs were obtained through a log-normal fit distribution yielding values of $1.3 \times 10^{-5} G_{0}$ and $2.2 \times 10^{-5} G_{0}$, respectively, where $G_{0}=2 e^{2} / h$ is the quantum of conductance, as shown in the corresponding one-dimensional (1D) conductance histograms (see Supporting Information (section 2.2)).

For the molecules with the meta-phenyl anchoring (ps-parameta-PCP and ps-meta-meta-PCP) the bias voltage in the transport experiment was increased to $250 \mathrm{mV}$ in order to bring molecular levels closer to resonance, as the conductance of these PCPs was below the detection limit for a bias of 100 $\mathrm{mV}$. However, even at this increased bias voltage, no clear conductance plateaus were detected, even when using the earlier-mentioned clustering method. Whether the lack of clear plateaus is due to molecular conductance below the detection threshold of the experimental setup of $10^{-6} G_{0}$ or the molecules' inability to form stable molecular junctions cannot be distinguished. The very short-breaking traces visible in Figure 2 at higher conductance values for both ps-para-metaand ps-meta-meta-PCP are most likely due to direct electron injection into the molecules' $\pi$-systems without controlled sulfur-to-sulfur immobilization.

Of particular interest are the distance-dependent singlemolecule junction charge-transport studies of ps-para-paraand ps-meta-para-PCPs, as substantial differences in the transport behavior under mechanical stress are expected between the central ps-para- and ps-meta-PCP subunits. While the mechanosensitivity of the ps-para-PCP subunit was already evidenced in modulation experiments for the pspara-para-OPE PCP (Figure 1$)^{28}$ and rationalized as being due to a distance-dependent conductance dip close to the Fermi level originating from DQI, similar behavior is not expected for the ps-meta-PCP subunit, as CQI has been predicted. ${ }^{29}$

To study the presence of DQI in more detail, modulation experiments are useful. The modulation procedure consists of constricting the gold wire down to a conductance of $2 G_{0}$ with the piezo control. ${ }^{28}$ The junction will then break by itself due to its surface tension. ${ }^{36}$ The electrodes are then separated by $7.5 \AA$, and a pulse of a triangular waveform with an amplitude of $20 \mathrm{~V}_{\text {peak-to-peak }}$ is applied to the piezoelectric element, which corresponds to a modulation amplitude of $5 \AA$ between the electrodes. The conductance is continuously monitored, and the modulation is applied at a frequency of $5 \mathrm{~Hz}$ for $15 \mathrm{~s}$. Hereafter, the junction is fused again, and a new modulation trace is recorded. The results of electrode-modulation experiments on PCPs $\mathbf{1}$ and $\mathbf{2}$ are summarized in Figure 3.

Similar to ps-para-para-OPE PCP, ${ }^{28}$ a rich variety of distance-modulated conductance responses has been observed for ps-para-para-PCP (1). The periodic electrode displacement caused a substantial conductance modulation, which was either in-phase (Figure 3a), anti-phase (Figure 3c), or even two times the frequency (Figure $3 \mathrm{~b}$ ) of the voltage applied to the piezo 
(a)

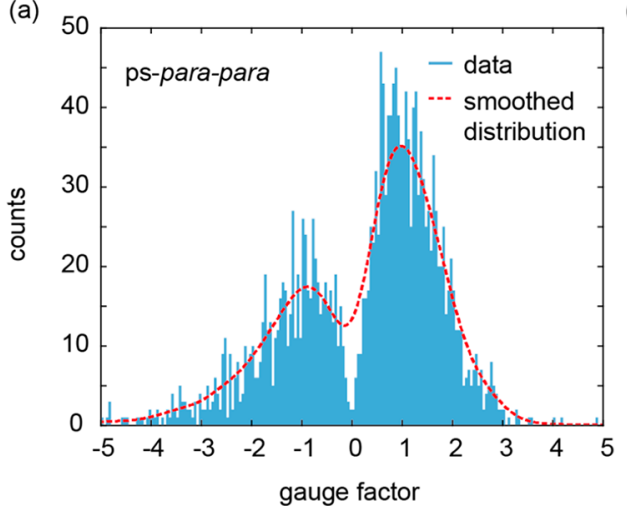

(b)

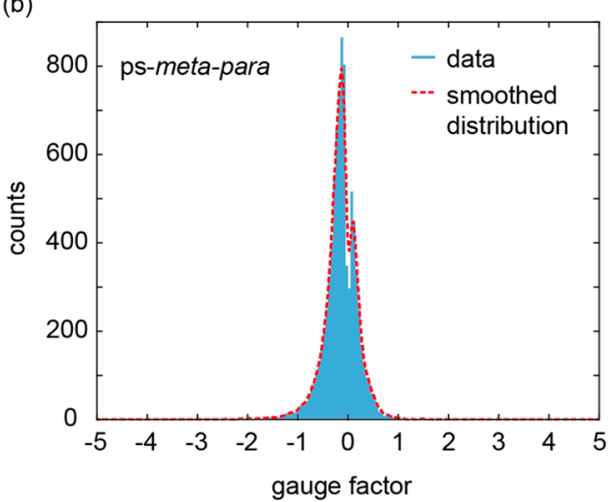

Figure 4. Gauge factors obtained from (a) 1442 ps-para-para-PCP traces and (b) 2236 ps-meta-para-PCP traces in modulation experiments. The counts are collected from fast Fourier transform (FFT) spectra over $1 \mathrm{~s}$ intervals of the traces, which means that there are 15 counts, with different GFs for each recording, lasting $15 \mathrm{~s}$.

(a)

ps-para-para PCP

ps-meta-para PCP
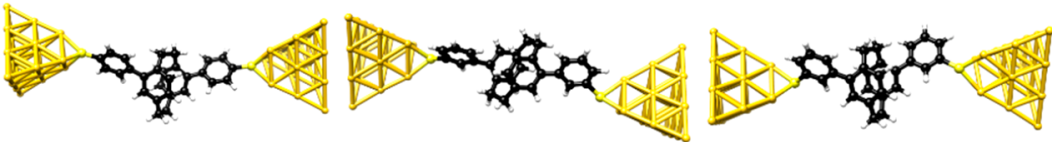

(b)

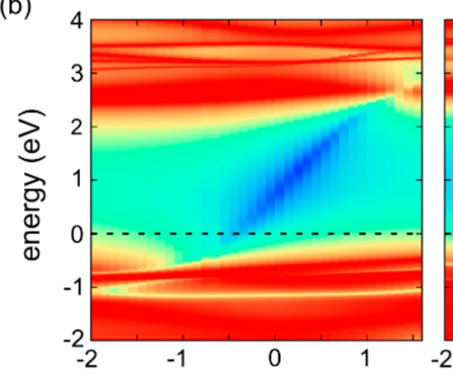

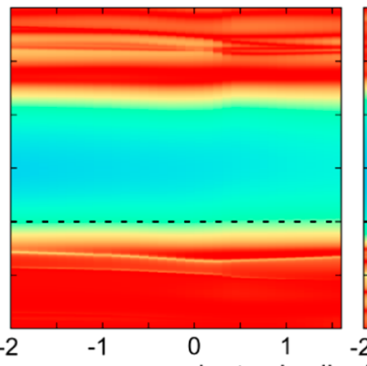

electrode displacement $(\AA)$
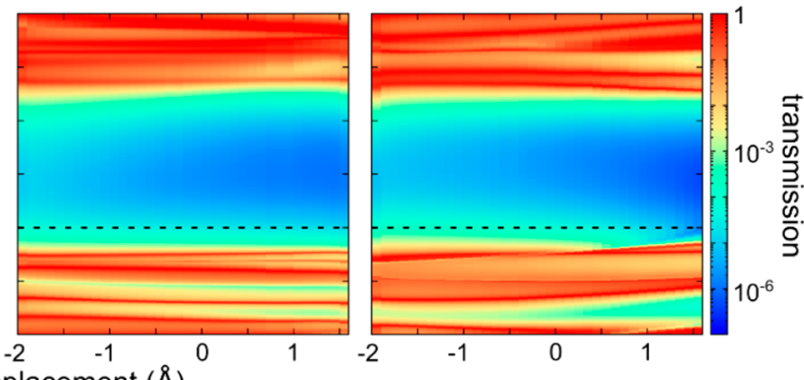

Figure 5. (a) Illustration of PCP derivatives 1-4 immobilized in junctions between two gold electrodes. (b) Transmission maps of the four types of PCP single-molecule junctions. The horizontal red resonances in the maps arise from molecular frontier orbitals. For the ps-para-para-PCP molecule, an antiresonance is observed inside the HOMO-LUMO gap that shifts in energy as the displacement is varied. Similar tunable DQI effects are absent for all other molecules, in particular for ps-meta-para-PCP junctions and ps-meta-meta-PCP junctions with central ps-meta-PCP systems. The position of the Fermi energy is indicated as a horizontal dashed line.

stack. As sketched in Figure 3e, this observed mechanosensitivity is caused by the DQI dip in the conductance versus molecular length relation (insets in Figure 3e), with the variety of observed behaviors reflecting the exact position of the conductance dip in the particular molecular junction. We take the in-phase case as an example to explain the observed behavior. This corresponds to a starting position in which the molecule is prestretched, as depicted in Figure 3e (top, light yellow background). In this case, whenever the molecule is stretched by increasing the voltage applied to the piezoelectric element, the conductance goes up, and the conductance goes down when the piezo voltage decreases, i.e., the conduction follows the applied piezo-voltage modulation in-phase.

The striking similarity of the mechanosensitivity of ps-parapara-PCP (1) and ps-para-para-OPE PCP, both comprising a central para-PCP subunit, not only points at this structural motif as the origin of the phenomenon but also further corroborates its rationalization based on the presence of a DQI dip in proximity of the Fermi level. Equally interesting are the modulation experiments performed with the ps-meta-para-PCP (2). As displayed in parts $\mathrm{d}$ and $\mathrm{f}$ of Figure 3, single-molecule junctions with this structure do not feature significant conductance changes during the modulation experiments. Remarkably, the absence of mechanosensitivity of the ps-metapara-PCP has not been reported experimentally before. Particularly, this behavior indicates the absence of a transmission dip as a function of electrode displacement (within the displacement window probed by the experiment), suggesting that DQI does not occur in the case of ps-meta-PCP cores. This is in agreement with previous predictions of $\pi$-stacked systems by the groups of Solomon ${ }^{26,27}$ and Yoshizawa. ${ }^{29}$

To quantify the mechanosensitivity of the molecule under investigation, the gauge factor (GF) was determined as the ratio between logarithmic conductance variation and linear electrode displacement (see the Supporting Information (section 2.4) for more information). Figure 4 displays the GFs for (a) ps-para-para-PCP and (b) ps-meta-para-PCP. Indeed, the GFs of the measurements of both structures visualize their difference. While the absence of mechanosensitivity of ps-meta-para-PCP results in a sharp peak close to zero (Figure 4b), the GF values recorded for ps-para-para-PCP 


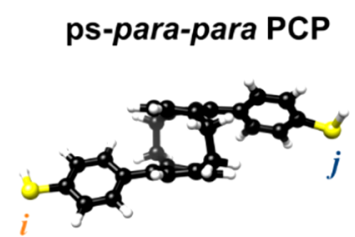

GPL

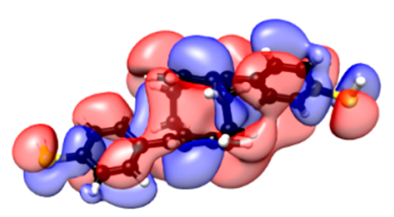

GPH

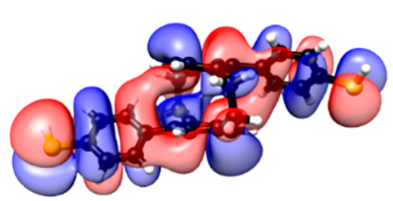

ps-meta-para PCP

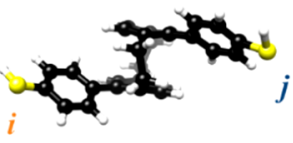

GPL

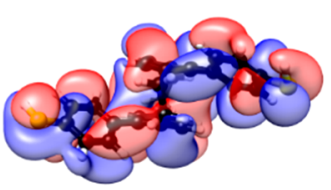

GPH

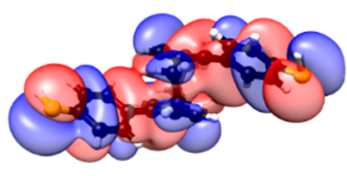

ps-para-meta PCP

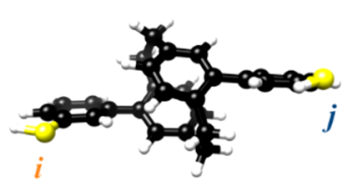

GPL

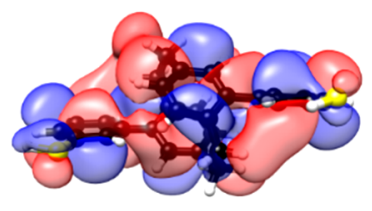

GPH

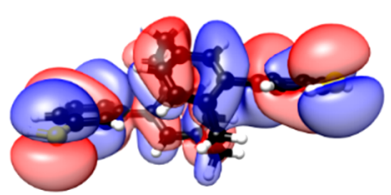

ps-meta-meta PCP

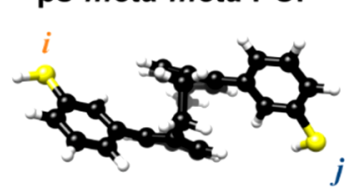

GPL
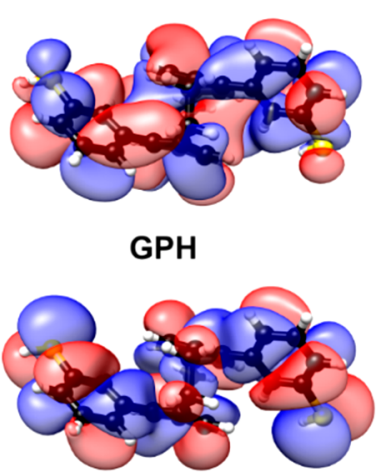

Figure 6. HOMO and LUMO molecular frontier orbitals of the ps-para-para-, ps-meta-para-, ps-para-meta-, and ps-meta-meta-PCPs. The anchoring sulfur atoms are marked $i$ and $j$, respectively. Extended representations also including GPH-1 and GPL +1 for each molecule can be found in the Supporting Information (section 3.3).

(Figure 4a) display a broad distribution with a minimum at GF $=0$, documenting its pronounced mechanosensitivity.

To rationalize the experimental observations described earlier, extensive DFT calculations were performed. First, the four model compounds ps-para-para-PCP (1b), ps-meta-paraPCP (2b), ps-para-meta-PCP (3b), and ps-meta-meta-PCP (4b) were optimized in the gas phase. The hydrogen atoms of the terminal thiol groups were removed, and the molecules were placed in model junctions, consisting of pairs of tetrahedral gold leads (Figure 5a). Optimizing the junction geometry, only the top three gold atoms in the first layer of each tip were allowed to relax, while the rest of the gold cluster remained fixed. Then, the systems were stretched in steps of $0.1 \AA$, and a geometry optimization was performed at every single step. A detailed description of the established approach $^{37}$ and explanations of the calculations are provided in the Supporting Information (section 3.1).

A closer look at the two-dimensional contour plots of transmission in dependence of energy and electrode displacement in Figure $5 \mathrm{~b}$ reveals important information about the transmission behavior of the molecular junctions inside the electronic gap between the highest occupied molecular orbital (HOMO) and the lowest unoccupied molecular orbital (LUMO). In the case of the ps-para-para-PCP, a transmission valley (blue diagonal trace) with transmission values lower than $10^{-5}$ is observed between the molecular frontier orbitals (red horizontal traces). The transmission valley corresponds to the DQI conductance dip, as shown in Figure 3e. The energy position of the transmission minimum can be tuned by mechanical manipulation of the junction. It should be noted that the DQI crosses the complete HOMO-LUMO gap, making it a robust feature for experimental detection, because it does not depend on the precise position of the Fermi energy. A similar valley is not present for the ps-meta-para-PCP junction. Instead, the transmission stays rather constant in the range of $\sim 10^{-3}-10^{-4}$ inside the molecule's HOMO-LUMO gap. Rather uniform transmission values are furthermore predicted in the molecules' electronic gap for both ps-para- meta- and ps-meta-meta-PCP. Interestingly, for this pair of model compounds with terminal meta-benzene linkers, $\sim 1$ order of magnitude lower transmission values were calculated compared to the pair with terminal para-benzene linkers. This is also in line with the absence of measurable conductance plateaus for the meta-phenyl-connected PCPs in Figure 2.

The conductance that we compute within the DFT-NEGF formalism $^{37}$ at the Fermi energy is plotted in parts e and $\mathrm{f}$ of Figure 3 for ps-para-para- and ps-meta-para-PCP derivatives, respectively. Considering the example of the ps-para-para-PCP single-molecule junction, the conductance features a dip that is shifted toward negative displacements and is $\sim 2$ orders of magnitude lower than the base value. Molecular contacts constructed from ps-meta-para-PCP show instead a rather constant behavior in the studied displacement range without a DQI dip.

The behavior of another geometry that features a more complex stick-slip motion is discussed in the Supporting Information (section 3.2). In that case, remnants of transmission valleys are visible for the ps-para-meta-PCP molecular junction, further consolidating the hypothesis of the central pspara-PCP subunit as the origin of the DQI phenomenon. Note, however, that we argue here on a qualitative level because we did not correct DFT quasiparticle energies and therefore expect uncertainties with respect to absolute conductance values, ${ }^{17,38-41}$ as a comparison of experimental and theoretical data in Figures 3 and 5 confirms.

While the calculated transmission plots perfectly support the hypothesis that mechanosensitivity can exclusively be observed for structures with a central ps-para-PCP subunit providing $\mathrm{DQI}$, another qualitative argument is provided by considering orbital symmetry rules, as suggested by Yoshizawa and coworkers. $^{29,42}$ The qualitative prediction of QI phenomena is based on the interplay of molecular frontier orbitals, especially the HOMO and LUMO, in transport models using LandauerBüttiker scattering theory and Green's function methods (details are provided in the Supporting Information (section 3.3)). Thus, the gas-phase frontier orbitals of the model 
compounds with terminal thiol groups were calculated and are displayed in Figure 6.

Assuming that the Fermi energy of the electrodes lies between the molecule's HOMO and LUMO energies due to charge neutrality, two orbital rules apply for the electronic transport properties. ${ }^{29,42}$ (1) The weights of the HOMO and LUMO wave functions on the anchoring atoms $i$ and $j$ (see Figure 6) need to be of decent size to yield a fair contribution to the transmission. (2) If the parities of the molecule's HOMO and LUMO on the anchoring atoms are different in sign, transport is symmetry-supported through CQI, which is typically reflected in a high transmission inside the HOMOLUMO gap. If HOMO and LUMO parities are the same instead, the related molecular orbital resonances cancel each other out at a certain energy inside the HOMO-LUMO gap. As a consequence, transport is symmetry-inhibited, resulting in a DQI dip in the energy-dependent transmission function, typically leading to a reduced conductance of the singlemolecule junction. This argument assumes that HOMO and LUMO orbital wave functions are of similar character on the termini $i$ and $j$.

The analysis of the molecules started by defining the terminal sulfur atoms as anchoring sites $i$ and $j$ (see Figure 6). Comparing orbital wave functions on the terminal sulfur atoms shows that gas-phase HOMO (GPH) and gas-phase LUMO (GPL) of both model compounds comprising a central paraPCP (ps-para-para- and ps-para-meta-PCP) are of similarly oriented $\pi$-character at the sulfur atoms and have the same parities; thus, these structures should show DQI. In contrast, both molecules with a ps-meta-PCP subunit have different parities in their GPH and GPL on the terminal sulfur atoms and thus exhibit CQI. The orbital symmetry rules thus rationalize the experimental observations and numerical computations reported earlier, which identify the central pspara-PCP subunit as the origin of DQI.

Let us point out that ferrocene recently emerged as a related $3 \mathrm{D}$ system to the PCP, where the angle between two cyclopentadienyl decks can be tuned rather continuously around the central $\mathrm{Fe}$ core atom. ${ }^{43}$ The torsion can be compared to meta or para connection to the PCP. The mechanical distortion that explains the experimental results here is mainly the displacement of two benzene rings as compared to a rotation.

\section{CONCLUSION}

We have studied the electronic transport properties of singlemolecule junctions based on $\pi$-stacked hydrocarbons. Our study confirms previous theoretical predictions for model compounds in terms of molecular orbital symmetry rules with regard to the suppression of electronic transport in PCP subunits when contacted in ps-para geometry as compared to ps-meta geometry. While the ps-meta subunit generally shows high conductance in comparison to the ps-para geometry (still relatively low compared to other conjugated molecular wires such as OPE3-dithiol ${ }^{44}$ ) and little sensitivity to mechanical manipulation, the ps-para subunit offers an exceptional mechanoelectric sensitivity. Notably, our theoretical calculations predict that the DQI can be tuned through the complete electronic gap region, explaining the experimental robustness of the feature, as observations should be largely independent of the precise location of the Fermi energy. Similar to an optical Fabry-Pérot interferometer, the DQI feature can be used to detect minute displacement changes and hence serves as a quantum sensor operating at ambient conditions, i.e., at room temperature.

\section{ASSOCIATED CONTENT}

\section{(s) Supporting Information}

The Supporting Information is available free of charge at https://pubs.acs.org/doi/10.1021/jacs.1c06966.

Synthesis and characterization of all molecules, and details concerning transport measurements and calculations (PDF)

\section{AUTHOR INFORMATION}

\section{Corresponding Authors}

Fabian Pauly - Institute of Physics, University of Augsburg, 86159 Augsburg, Germany; (1) orcid.org/0000-0001-80172379; Email: fabian.pauly@uni-a.de

Herre S. J. van der Zant - Kavli Institute of Nanoscience, Delft University of Technology, 2628 GJ Delft, The Netherlands; (1) orcid.org/0000-0002-5385-0282; Email: h.s.j.vanderzant@tudelft.nl

Marcel Mayor - Department of Chemistry, University of Basel, 4056 Basel, Switzerland; Institute for Nanotechnology, Karlsruhe Institute of Technology (KIT), 76021 Karlsruhe, Germany; Lehn Institute of Functional Materials, School of Chemistry, Sun Yat-Sen University, Guangzhou 510274, P.

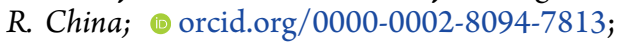
Email: marcel.mayor@unibas.ch

\section{Authors}

Ksenia Reznikova - Department of Chemistry, University of Basel, 4056 Basel, Switzerland

Chunwei Hsu - Kavli Institute of Nanoscience, Delft University of Technology, 2628 GJ Delft, The Netherlands

Werner M. Schosser - Institute of Physics, University of Augsburg, 86159 Augsburg, Germany

Almudena Gallego - Department of Chemistry, University of Basel, 4056 Basel, Switzerland

Katawoura Beltako - Institute of Physics, University of

Augsburg, 86159 Augsburg, Germany

Complete contact information is available at:

https://pubs.acs.org/10.1021/jacs.1c06966

\section{Author Contributions \\ ${ }^{\#}$ K.R., C.H., and W.M.S. contributed equally. \\ Notes}

The authors declare no competing financial interest.

\section{ACKNOWLEDGMENTS}

This work was supported by a Horizon 2020 FET open project (QuIET; no. 767187). H.S.J.v.d.Z. and C.H. acknowledge support from The Netherlands Organisation for Scientific Research (NWO-Vrij program). M.M. acknowledges support from the Swiss National Science Foundation (SNF Grant no. 200020-178808) and the 111 project (90002-18011002).

\section{ABBREVIATIONS}

$\mathrm{QI}$, quantum interference; DQI, destructive quantum interference; CQI, constructive quantum interference; OPE, oligo(phenylene ethynylene); PCP, [2.2]paracyclophane; MCBJ, mechanically controlled break junction; DFT, density functional theory; NEGF, nonequilibrium Green's function; ps, pseudo; GF, gauge factor; FFT, fast Fourier transform; 
HOMO, highest occupied molecular orbital; LUMO, lowest unoccupied molecular orbital; GPH, gas-phase HOMO; GPL, gas-phase LUMO.

\section{REFERENCES}

(1) van der Molen, S. J.; Naaman, R.; Scheer, E.; Neaton, J. B.; Nitzan, A.; Natelson, D.; Tao, N. J.; van der Zant, H. S. J.; Mayor, M.; Ruben, M.; Reed, M.; Calame, M. Visions for a Molecular Future. Nat. Nanotechnol. 2013, 8 (6), 385-389.

(2) Aviram, A.; Ratner, M. A. Molecular Rectifiers. Chem. Phys. Lett. 1974, 29 (2), 277-283.

(3) Luo, L.; Choi, S. H.; Frisbie, C. D. Probing Hopping Conduction in Conjugated Molecular Wires Connected to Metal Electrodes. Chem. Mater. 2011, 23 (3), 631-645.

(4) Huber, R.; González, M. T.; Wu, S.; Langer, M.; Grunder, S.; Horhoiu, V.; Mayor, M.; Bryce, M. R.; Wang, C.; Jitchati, R.; Schönenberger, C.; Calame, M. Electrical Conductance of Conjugated Oligomers at the Single Molecule Level. J. Am. Chem. Soc. 2008, 130 (3), 1080-1084.

(5) Irie, M.; Fukaminato, T.; Sasaki, T.; Tamai, N.; Kawai, T. A Digital Fluorescent Molecular Photoswitch. Nature 2002, 420 (6917), 759-760.

(6) Liao, J.; Agustsson, J. S.; Wu, S.; Schönenberger, C.; Calame, M.; Leroux, Y.; Mayor, M.; Jeannin, O.; Ran, Y.-F.; Liu, S.-X.; Decurtins, S. Cyclic Conductance Switching in Networks of Redox-Active Molecular Junctions. Nano Lett. 2010, 10 (3), 759-764.

(7) Elbing, M.; Ochs, R.; Koentopp, M.; Fischer, M.; von Hanisch, C.; Weigend, F.; Evers, F.; Weber, H. B.; Mayor, M. A SingleMolecule Diode. Proc. Natl. Acad. Sci. U. S. A. 2005, 102 (25), 88158820 .

(8) Lörtscher, E.; Gotsmann, B.; Lee, Y.; Yu, L.; Rettner, C.; Riel, H. Transport Properties of a Single-Molecule Diode. ACS Nano 2012, 6 (6), 4931-4939.

(9) Naher, M.; Milan, D. C.; Al-Owaedi, O. A.; Planje, I. J.; Bock, S.; Hurtado-Gallego, J.; Bastante, P.; Abd Dawood, Z. M.; Rincón-García, L.; Rubio-Bollinger, G.; Higgins, S. J.; Agraï, N.; Lambert, C. J.; Nichols, R. J.; Low, P. J. Molecular Structure-(Thermo)Electric Property Relationships in Single-Molecule Junctions and Comparisons with Single- and Multiple-Parameter Models. J. Am. Chem. Soc. 2021, 143 (10), 3817-3829.

(10) Stadler, R.; Markussen, T. Controlling the Transmission Line Shape of Molecular T-Stubs and Potential Thermoelectric Applications. J. Chem. Phys. 2011, 135 (15), 154109.

(11) Markussen, T.; Stadler, R.; Thygesen, K. S. The Relation between Structure and Quantum Interference in Single Molecule Junctions. Nano Lett. 2010, 10 (10), 4260-4265.

(12) Jiang, F.; Trupp, D. I.; Algethami, N.; Zheng, H.; He, W.; Alqorashi, A.; Zhu, C.; Tang, C.; Li, R.; Liu, J.; Sadeghi, H.; Shi, J.; Davidson, R.; Korb, M.; Sobolev, A. N.; Naher, M.; Sangtarash, S.; Low, P. J.; Hong, W.; Lambert, C. J. Turning the Tap: Conformational Control of Quantum Interference to Modulate Single-Molecule Conductance. Angew. Chem., Int. Ed. 2019, 58 (52), 18987-18993.

(13) Venkataraman, L.; Klare, J. E.; Nuckolls, C.; Hybertsen, M. S.; Steigerwald, M. L. Dependence of Single-Molecule Junction Conductance on Molecular Conformation. Nature 2006, 442 (7105), 904-907.

(14) Aradhya, S. V.; Meisner, J. S.; Krikorian, M.; Ahn, S.; Parameswaran, R.; Steigerwald, M. L.; Nuckolls, C.; Venkataraman, L. Dissecting Contact Mechanics from Quantum Interference in SingleMolecule Junctions of Stilbene Derivatives. Nano Lett. 2012, 12 (3), $1643-1647$.

(15) Mayor, M.; Weber, H. B.; Reichert, J.; Elbing, M.; von Hänisch, C.; Beckmann, D.; Fischer, M. Electric Current through a Molecular Rod-Relevance of the Position of the Anchor Groups. Angew. Chem., Int. Ed. 2003, 42 (47), 5834-5838.

(16) Koga, J.; Tsuji, Y.; Yoshizawa, K. Orbital Control of SingleMolecule Conductance Perturbed by $\pi$-Accepting Anchor Groups:
Cyanide and Isocyanide. J. Phys. Chem. C 2012, 116 (38), 2060720616.

(17) Arroyo, C. R.; Tarkuc, S.; Frisenda, R.; Seldenthuis, J. S.; Woerde, C. H. M.; Eelkema, R.; Grozema, F. C.; van der Zant, H. S. J. Signatures of Quantum Interference Effects on Charge Transport Through a Single Benzene Ring. Angew. Chem., Int. Ed. 2013, 52 (11), 3152-3155.

(18) Kiguchi, M.; Kaneko, S. Single Molecule Bridging between Metal Electrodes. Phys. Chem. Chem. Phys. 2013, 15 (7), 2253-2267.

(19) Lambert, C. J. Basic Concepts of Quantum Interference and Electron Transport in Single-Molecule Electronics. Chem. Soc. Rev. 2015, 44 (4), 875-888.

(20) Kiguchi, M.; Nakamura, H.; Takahashi, Y.; Takahashi, T.; Ohto, T. Effect of Anchoring Group Position on Formation and Conductance of a Single Disubstituted Benzene Molecule Bridging Au Electrodes: Change of Conductive Molecular Orbital and Electron Pathway. J. Phys. Chem. C 2010, 114 (50), 22254-22261.

(21) Arroyo, C. R.; Frisenda, R.; Moth-Poulsen, K.; Seldenthuis, J. S.; Bjørnholm, T.; van der Zant, H. S. Quantum Interference Effects at Room Temperature in OPV-Based Single-Molecule Junctions. Nanoscale Res. Lett. 2013, 8, 1.

(22) Li, X.; Staykov, A.; Yoshizawa, K. Orbital Views of the Electron Transport through Polycyclic Aromatic Hydrocarbons with Different Molecular Sizes and Edge Type Structures. J. Phys. Chem. C 2010, 114 (21), 9997-10003.

(23) Yoshizawa, K.; Tada, T.; Staykov, A. Orbital Views of the Electron Transport in Molecular Devices. J. Am. Chem. Soc. 2008, 130 (29), 9406-9413.

(24) Yoshizawa, K. An Orbital Rule for Electron Transport in Molecules. Acc. Chem. Res. 2012, 45 (9), 1612-1621.

(25) Brown, C. J.; Farthing, A. C. Preparation and Structure of Di- $p$ -Xylylene. Nature 1949, 164 (4178), 915-916.

(26) Solomon, G. C.; Vura-Weis, J.; Herrmann, C.; Wasielewski, M. R.; Ratner, M. A. Understanding Coherent Transport through $\pi$ Stacked Systems upon Spatial Dislocation. J. Phys. Chem. B 2010, 114 (45), 14735-14744.

(27) Solomon, G. C.; Herrmann, C.; Vura-Weis, J.; Wasielewski, M. R.; Ratner, M. A. The Chameleonic Nature of Electron Transport through $\pi$-Stacked Systems. J. Am. Chem. Soc. 2010, 132 (23), 78877889.

(28) Stefani, D.; Weiland, K. J.; Skripnik, M.; Hsu, C.; Perrin, M. L.; Mayor, M.; Pauly, F.; van der Zant, H. S. J. Large Conductance Variations in a Mechanosensitive Single-Molecule Junction. Nano Lett. 2018, 18 (9), 5981-5988.

(29) Li, X.; Staykov, A.; Yoshizawa, K. Orbital Views on ElectronTransport Properties of Cyclophanes: Insight into Intermolecular Transport. Bull. Chem. Soc. Jpn. 2012, 85 (2), 181-188.

(30) Perdew, J. P. Density-Functional Approximation for the Correlation Energy of the Inhomogeneous Electron Gas. Phys. Rev. B: Condens. Matter Mater. Phys. 1986, 33 (12), 8822-8824.

(31) Vorontsova, N. V.; Rozenberg, V. I.; Sergeeva, E. V.; Vorontsov, E. V.; Starikova, Z. A.; Lyssenko, K. A.; Hopf, H. Symmetrically Tetrasubstituted [2.2]Paracyclophanes: Their Systematization and Regioselective Synthesis of Several Types of Bis-Bifunctional Derivatives by Double Electrophilic Substitution. Chem. - Eur. J. 2008, 14 (15), 4600-4617.

(32) Jevric, M.; Petersen, A. U.; Mansø, M.; Madsen, A. Ø.; Nielsen, M. B. Bismuth(III)-Promoted Acetylation of Thioethers into Thioacetates. Eur. J. Org. Chem. 2015, 2015, 4675-4688.

(33) Martin, C. A.; Ding, D.; van der Zant, H. S. J.; van Ruitenbeek, J. M. Lithographic Mechanical Break Junctions for Single-Molecule Measurements in Vacuum: Possibilities and Limitations. New J. Phys. 2008, 10 (6), 065008.

(34) Martin, C. A.; Smit, R. H. M.; van Egmond, R.; van der Zant, H. S. J.; van Ruitenbeek, J. M. A Versatile Low-Temperature Setup for the Electrical Characterization of Single-Molecule Junctions. Rev. Sci. Instrum. 2011, 82 (5), 053907.

(35) Cabosart, D.; El Abbassi, M.; Stefani, D.; Frisenda, R.; Calame, M.; van der Zant, H. S. J.; Perrin, M. L. A Reference-Free Clustering 
Method for the Analysis of Molecular Break-Junction Measurements. Appl. Phys. Lett. 2019, 114 (14), 143102.

(36) O’Neill, K.; Osorio, E. A.; van der Zant, H. S. J. Self-Breaking in Planar Few-Atom Au Constrictions for Nanometer-Spaced Electrodes. Appl. Phys. Lett. 2007, 90 (13), 133109.

(37) Pauly, F.; Viljas, J. K.; Huniar, U.; Häfner, M.; Wohlthat, S.; Bürkle, M.; Cuevas, J. C.; Schön, G. Cluster-Based Density-Functional Approach to Quantum Transport through Molecular and Atomic Contacts. New J. Phys. 2008, 10 (12), 125019.

(38) Quek, S. Y.; Venkataraman, L.; Choi, H. J.; Louie, S. G.; Hybertsen, M. S.; Neaton, J. B. Amine-Gold Linked Single-Molecule Circuits: Experiment and Theory. Nano Lett. 2007, 7 (11), 34773482.

(39) Zotti, L. A.; Bürkle, M.; Pauly, F.; Lee, W.; Kim, K.; Jeong, W.; Asai, Y.; Reddy, P.; Cuevas, J. C. Heat Dissipation and Its Relation to Thermopower in Single-Molecule Junctions. New J. Phys. 2014, 16 (1), 015004.

(40) Manrique, D. Z.; Huang, C.; Baghernejad, M.; Zhao, X.; AlOwaedi, O. A.; Sadeghi, H.; Kaliginedi, V.; Hong, W.; Gulcur, M.; Wandlowski, T.; Bryce, M. R.; Lambert, C. J. A Quantum Circuit Rule for Interference Effects in Single-Molecule Electrical Junctions. Nat. Commun. 2015, 6 (1), 6389.

(41) Garner, M. H.; Li, H.; Chen, Y.; Su, T. A.; Shangguan, Z.; Paley, D. W.; Liu, T.; Ng, F.; Li, H.; Xiao, S.; Nuckolls, C.; Venkataraman, L.; Solomon, G. C. Comprehensive Suppression of Single-Molecule Conductance Using Destructive $\sigma$-Interference. Nature 2018, 558 (7710), 415-419.

(42) Tsuji, Y.; Yoshizawa, K. Frontier Orbital Perspective for Quantum Interference in Alternant and Nonalternant Hydrocarbons. J. Phys. Chem. C 2017, 121 (17), 9621-9626.

(43) Camarasa-Gómez, M.; Hernangómez-Pérez, D.; Inkpen, M. S.; Lovat, G.; Fung, E.-D.; Roy, X.; Venkataraman, L.; Evers, F. Mechanically Tunable Quantum Interference in Ferrocene-Based Single-Molecule Junctions. Nano Lett. 2020, 20 (9), 6381-6386.

(44) Frisenda, R.; Perrin, M. L.; Valkenier, H.; Hummelen, J. C.; van der Zant, H. S. J. Statistical Analysis of Single-Molecule Breaking Traces. Phys. Status Solidi B 2013, 250 (11), 2431-2436. 